\title{
Forensic Science in the Pharmaceutical Industry - A Microscopy Perspective
}

\author{
J. A. Reffner,
}

\author{
John Jay College, CUNY, 445 W $59^{\text {th }}$ Street, New York, NY10019
}

Forensic science is an evolving but critical discipline within the pharmaceutical industry. Due to the amount of sample available for analysis, microscopy and microanalytical techniques are the basis for much of the testing performed to establish and protect intellectual property, maintain quality assurance throughout a drug product's life cycle and support various other requirements of scientists working within this highly regulated industry. In recent years, the infiltration of counterfeit pharmaceuticals into the legitimate supply chain has created a new area of forensic science within the pharmaceutical industry. The need to differentiate authentic from counterfeit goods in both the laboratory and remotely has become a significant focus for brand owners and generic companies alike. Development of methods to differentiate these items is a significant challenge and typically requires the use of microscopy and microanalytical techniques.

Counterfeit sourcing and detection, though, is only one of many disciplines within forensic science that are widely applied within the pharmaceutical industry. The job title "Forensic Scientist" is becoming more and more common within the pharmaceutical industry. Forensic scientists rely upon microscopy and microanalytical methods and are using these methods to solve and support complex industry problems including intellectual property protection, analysis of, and tracing the source of contaminants and other types of quality assurance testing.

Figure 1 shows an overlay of infrared spectra collected from two different solid-state forms of the same active pharmaceutical ingredient (API); one is a spectrum of the crystalline form and the other is a spectrum of the amorphous form. Analysis was performed on microscopic quantities of sample, but provides court-admissible scientific evidence identifying these two forms. Aside from the impact on intellectual property rights, these different forms may also exhibit different bioavailability and/or have different physical and chemical properties that can be tailored to improve the product or its manufacturing process.

In a second example, shown in Figure 2, infrared spectra collected from packaging used in authentic and replicate samples of the same drug product are reported. Packaging is frequently used to differentiate authentic samples from counterfeits. Using a light microscope with infrared spectrometer, analyses are performed in seconds without sample destruction. Similar analysis could also be performed using a light microscope with a Raman microprobe. SEM-EDX analysis is also useful in product differentiation.

Figure 3 shows a photomicrograph of covert markers embedded within an electronic circuit board. Using alternate-wavelength light sources, these markers are easily located and detected making it possible to easily and reliably differentiate authentic from counterfeit articles. When used within the pharmaceutical industry, these markers are made from GRAS-approved materials that are easily incorporated within the raw materials, drug product or packaging. "GRAS" is an acronym for Generally Recognized As Safe. Sections 201(s) and 409 of the Federal Food, Drug, and Cosmetic Act states any substance that is intentionally added to food is a food additive unless the substance is 
generally recognized, among qualified experts, as having been adequately shown to be safe under the conditions of its intended use.

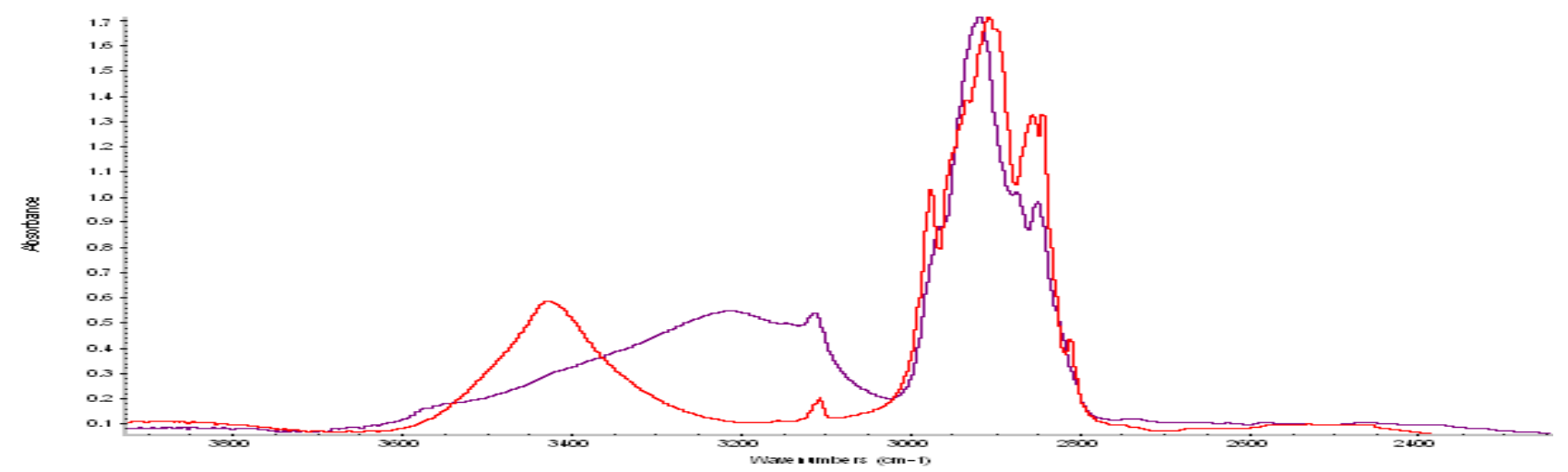

Figure 1. Comparison of Infrared Differences Due to Differences in Physical Form

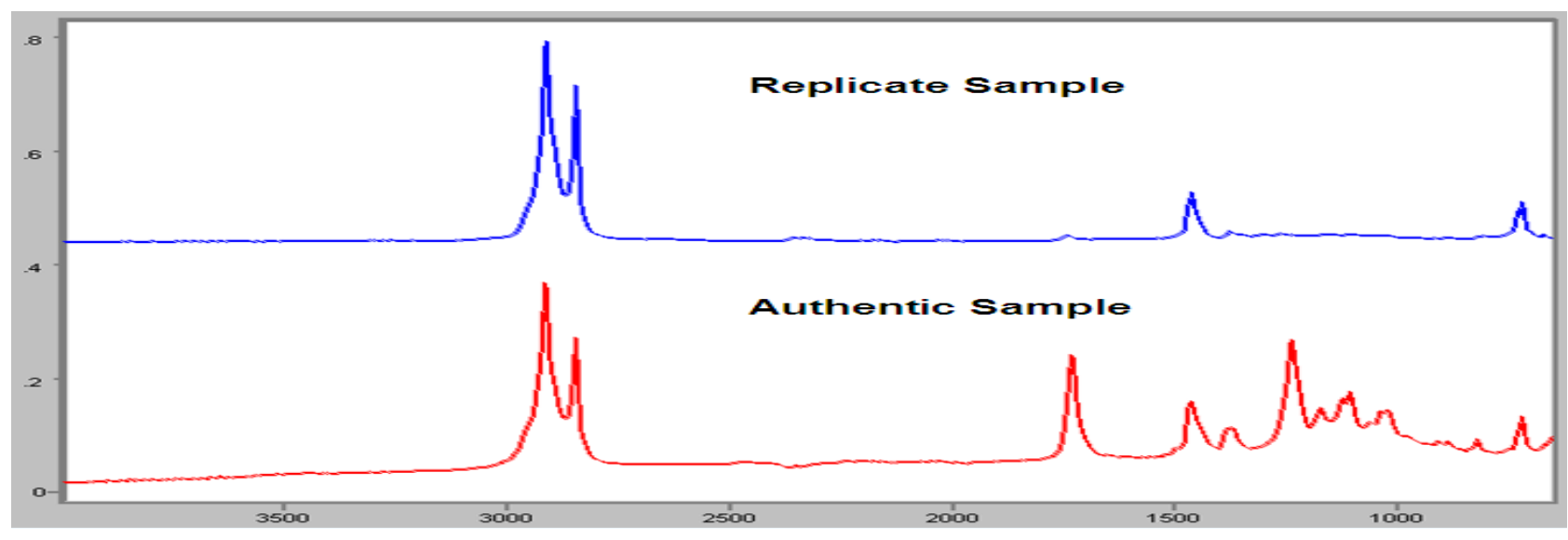

Figure 2. Infrared Spectra from Packaging of Authentic and Replicate Drug Product

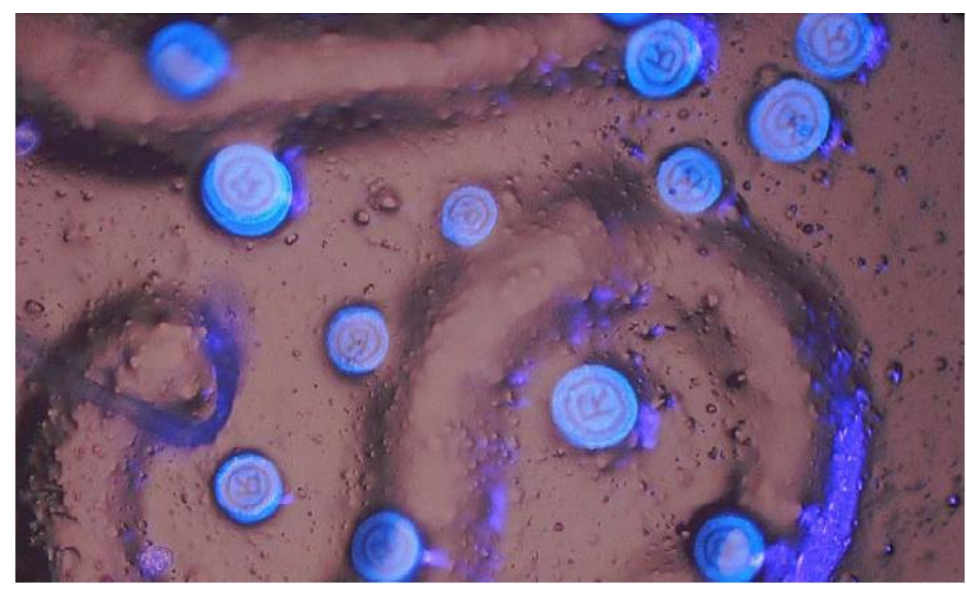

Figure 3. Photomicrograph of Covert Markers Embedded in an Electronic Circuit Board 